\title{
Interaction-disorder competition in a spin system evaluated through the Loschmidt Echo
}

\author{
Pablo R. Zangara, ${ }^{1}$ Axel D. Dente, ${ }^{1,2}$ Aníbal Iucci, ${ }^{3}$ Patricia R. Levstein, ${ }^{1}$ and Horacio M. Pastawski ${ }^{1}$ \\ ${ }^{1}$ Instituto de Física Enrique Gaviola (IFEG), CONICET-UNC and Facultad de Matemática, \\ Astronomía y Física, Universidad Nacional de Córdoba, 5000 Córdoba, Argentina \\ ${ }^{2}$ INVAP S.E., 8403 San Carlos de Bariloche, Argentina \\ ${ }^{3}$ Instituto de Física La Plata (IFLP), CONICET-UNLP and Departamento de Física, \\ Universidad Nacional de La Plata, CC 67, 1900 La Plata, Argentina
}

\begin{abstract}
The interplay between interactions and disorder in closed quantum many-body systems is relevant for thermalization phenomenon. In this article, we address this competition in an infinite temperature spin system, by means of the Loschmidt echo (LE), which is based on a time reversal procedure. This quantity has been formerly employed to connect quantum and classical chaos, and in the present many-body scenario we use it as a dynamical witness. We assess the LE time scales as a function of disorder and interaction strengths. The strategy enables a qualitative phase diagram that shows the regions of ergodic and nonergodic behavior of the polarization under the echo dynamics.

PACS numbers: 71.30.+h,03.65.Yz,72.15.Rn,05.70.Ln
\end{abstract}

The ergodic hypothesis of statistical mechanics implies the equivalence between time and ensemble averages. It is expected that a conservative many-body system satisfying such hypothesis would explore uniformly the whole energy shell. It is now a long time since Fermi, Pasta and Ulam (FPU) $\underline{1}$ questioned how the irregular dynamics induced by nonlinearities in a Hamiltonian may lead to energy equipartition as a manifestation of ergodicity. Such dynamics, referred to as thermalization, did not show up in their pioneering numerical simulations. Even though their striking results are now explained by the theory of $\operatorname{chaos}^{2}$, the solution of the quantum analogues are still in the early stages $3 . \underline{3}$.

Thermalization in isolated, strongly interacting, quantum systems is defined relative to a certain set of observables ${ }^{5}$. In particular, remarkable experiments have been performed to monitor momentum distributions of cold atoms loaded in one-dimensional optical lattices ${ }^{6}$, where the integrability of the underlying dynamics is weakly broken. Accordingly, a fundamental question is whether a nonergodic to ergodic transition threshold exists as one may go parametrically from an integrable to a nonintegrable quantum system. In the FPU problem one can surely answer affirmatively, since the onset of dynamical chaos can play the role for such a transition 2 . For interacting quantum systems in the presence of disorder, it is expected that a phase transition exists between delocalized states and a phase characterized by many-body localization $(\mathrm{MBL})^{7}$. This would constitute the sought threshold between ergodic and nonergodic behavior ${ }^{4}$.

The MBL results from a quantum dynamical phase transition between diffusive (ergodic) and localized (nonergodic) dynamics 8.9 . It occurs at nonzero (and eventually infinite) temperature, and is manifested in dynamical properties. The crucial idea is a competition between interactions and Anderson disorder ${ }^{10}$. In the many-body ergodic phase, the expectation values of observables computed on a finite subsystem, using a sin- gle energy eigenstate of the whole interacting system, would coincide with those evaluated in the corresponding microcanonical thermal ensemble $\mathrm{e}^{\underline{11}, 12}$. In this condition one may say that the system acts as its own heat bath. Quite on the contrary, in the nonergodic manybody phase, dynamics resembles a "glassy" behavior that precludes self-thermalization. In view of the difficulties of addressing a full many-body dynamics of specific correlation functions ${ }^{13,14}$, much of the progress in assessing the transition between the mentioned regimes relied mainly on the evaluation of spectral properties ${ }^{9,15}$.

The dynamics of specific observables has proved useful to monitor the onset of many-body chaos 16 . In particular, the Survival Probability (SP) of the eigenstates of an unperturbed Hamiltonian $\hat{H}_{0}$ under the evolution of the full interacting Hamiltonian $\hat{H}_{0}+\hat{\Sigma}$ decays with a characteristic time scale $\tau$. In such a case, a crossover from an exponential controlled by $1 / \tau \propto\|\hat{\Sigma}\|^{2}$ to a Gaussian characterized by $1 / \tau \propto\|\hat{\Sigma}\|$ is interpreted as evidence of the onset of a chaotic structure in the eigenstates of $\hat{H}_{0}+\hat{\Sigma}^{17-19}$. The first regime is described by the Fermi golden rule (FGR), and hence the breakdown of the FGR leads to dynamical chaos. Much in the spirit of this dynamical approach, in this article we propose and analyze the evolution of an experimentally accessible local observable, as a way to assess the interaction-disorder competition in a many-spin system. We resort to the Loschmidt echo $(\mathrm{LE})^{20}$, a measure of the revival that occurs when a time-reversal procedure is applied to $\hat{H}_{0}$. The LE has been experimentally evaluated from the local polarization of spin systems to quantify the role of perturbations (i.e. $\hat{\Sigma}$ ) and the system's own complexity on dynamical reversibility ${ }^{21}$. Besides, in classically chaotic systems, it is well-known that the LE of a semiclassical excitation undergoes a transition into a regime where its decay rate is given by the classical Lyapunov exponent ${ }^{22}$. Here, we use the LE dynamics as a time-dependent au- 
tocorrelation function that quantifies the time scales and ergodicity. Specifically, the time reversal procedure "filters out" the irrelevant dynamics produced by an integrable $\hat{H}_{0}$ which would hide the sought information ${ }^{23}$. Thus, the LE becomes a privileged dynamical witness of the competition between the interactions and disorder and a potential experimental candidate.

The spin model.- We consider a spin chain, whose dynamics is given by the total Hamiltonian $\hat{H}=\hat{H}_{0}+\hat{\Sigma}$ :

$$
\begin{gathered}
\hat{H}_{0}=\sum_{i=1}^{N} J\left[S_{i}^{x} S_{i+1}^{x}+S_{i}^{y} S_{i+1}^{y}\right] \\
\hat{\Sigma}=\sum_{i=1}^{N} \Delta S_{i}^{z} S_{i+1}^{z}+\sum_{i=1}^{N} h_{i} S_{i}^{z}
\end{gathered}
$$

where $\Delta$ is the magnitude of the homogeneous Ising interaction and $h_{i}$ are randomly distributed fields in an interval $[-h, h]$. Periodic boundary conditions (ring) are imposed and, unless explicitly stated, $N=12$. Notice that $\hat{H}_{0}$ can be mapped into two independent noninteracting fermion systems by the Wigner-Jordan transformation ${ }^{24}$, while $\hat{\Sigma}$ includes both the two-body Ising interaction and the local fields (disorder). Since $\hat{H}_{0}$ encloses single-particle physics, we consider it as the irrelevant part of the dynamics, a term that the LE manages to get rid of, allowing us to focus on interactions and disorder. This fact justifies the idea of using the LE as a filter for the relevant physical processes.

The spin autocorrelation function evaluated as a (local) Loschmidt echo.- We consider a high (infinite) temperature state formally denoted by $\left|\Psi_{e q}\right\rangle$, which represents an ensemble average over all basis states with the same statistical weights. We study the spin autocorrelation function at the particular site 1 ,

$$
M_{1,1}\left(2 t_{R}\right)=\frac{\left\langle\Psi_{e q}\left|\hat{S}_{1}^{z}\left(2 t_{R}\right) \hat{S}_{1}^{z}(0)\right| \Psi_{e q}\right\rangle}{\left\langle\Psi_{e q}\left|\hat{S}_{1}^{z}(0) \hat{S}_{1}^{z}(0)\right| \Psi_{e q}\right\rangle}
$$

where the spin operator is written in the Heisenberg picture as:

$$
\hat{S}_{1}^{z}\left(2 t_{R}\right)=\hat{U}_{+}^{\dagger}\left(t_{R}\right) \hat{U}_{-}^{\dagger}\left(t_{R}\right) \hat{S}_{1}^{z} \hat{U}_{-}\left(t_{R}\right) \hat{U}_{+}\left(t_{R}\right) .
$$

The evolution operators are $\hat{U}_{+}\left(t_{R}\right)=\exp \left[-\frac{\mathrm{i}}{\hbar}\left(\hat{H}_{0}+\right.\right.$ $\left.\hat{\Sigma}) t_{R}\right]$ and $\hat{U}_{-}\left(t_{R}\right)=\exp \left[-\frac{\mathrm{i}}{\hbar}\left(-\hat{H}_{0}+\hat{\Sigma}\right) t_{R}\right]$. Therefore, it is explicit that the echo procedure performed over $\hat{H}_{0}$ yields a global evolution operator $\hat{U}\left(2 t_{R}\right)=$ $\hat{U}_{-}\left(t_{R}\right) \hat{U}_{+}\left(t_{R}\right)$. Using cyclic invariance of the trace, one can replace the average over all basis states by an average over all states that have spin 1 up-polarized ${ }^{23}$. Additionally, we replace the ensemble average by an average over a few entangled states 25 , which provides a quadratic speedup of computational efforts. It yields equivalent results provided that only local observables (e.g. Eq. 3)
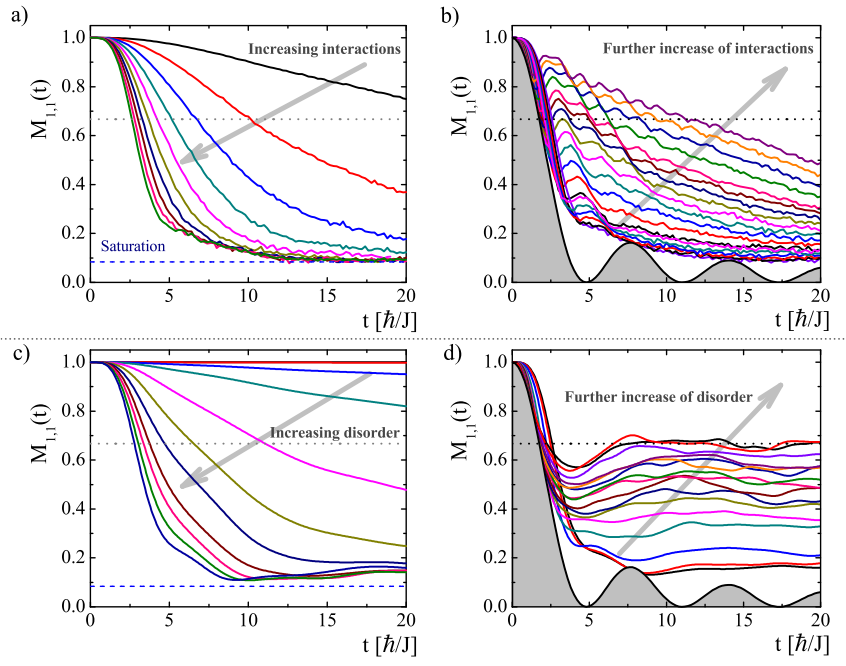

FIG. 1. Color online. Loschmidt echo for different parameter regimes. The characteristic time scale is defined by the decay up to $2 / 3$, shown by the horizontal dotted line. (a) $h=0$, $0<\Delta \lesssim 1.5 J$. Smooth decay, until saturation is reached (horizontal dashed line). (b) $h=0,1.5 J<\Delta<4.8 J$. LE long tails destroys saturation. The SP given by Eq. 7 determines the limit time scale (shaded region). (c) $\Delta=0$, $0<h \lesssim 1.0 \mathrm{~J}$. The localization length remains bigger than the system size. (d) $\Delta=0,1.0 \mathrm{~J}<h<5.5 \mathrm{~J}$. The localization length turns to be smaller than the system's size, and the polarization keeps around site 1 .

are evaluated. Thus, we consider:

$$
\left|\Psi_{n e q}\right\rangle=\left|\uparrow_{1}\right\rangle \otimes\left\{\sum_{r=1}^{2^{N-1}} \frac{1}{\sqrt{2^{N-1}}} e^{i \varphi_{r}}\left|\beta_{r}\right\rangle\right\}
$$

where $\varphi_{r}$ is a random phase and $\left\{\left|\beta_{r}\right\rangle\right\}$ are state vectors in the computational Ising basis of the $N-1$ remaining spins. The $M_{1,1}$ is now written in the Schrödinger picture as:

$$
M_{1,1}(2 t)=2\left\langle\Psi_{n e q}\left|\hat{U}_{+}^{\dagger}(t) \hat{U}_{-}^{\dagger}(t) \hat{S}_{1}^{z} \hat{U}_{-}(t) \hat{U}_{+}(t)\right| \Psi_{n e q}\right\rangle
$$

The explicit time dependence of $M_{1,1}(2 t)$ is evaluated by means of a fourth order Trotter-Suzuki decomposition without any Hilbert space truncation, implemented on general purpose graphical processing units 26 .

Results.- We start the evaluation of Eq. 6] with $h=0$ (no disorder), increasing the interaction strength $\Delta$ from zero, see Fig. 1 (a) and (b). The LE short-time scaling is $1-M_{1,1}(t) \propto t^{4}$, which can be analytically verified by expanding the evolution operators up to fourth order. Beyond the short-time window, it has a smooth decay produced by the nonreversed terms $\hat{\Sigma}^{23}$. As we do not have an explicit functional form for this regime, we define an effective characteristic time $\tau$ for the plotted curves as the decay up to $2 / 3$. The rates $1 / \tau$ are plotted in Fig. 2 as function of $\Delta$, for different disorder magnitudes $h$. 
Beyond the decay regime, as shown in Fig. 1-(a), the LE saturates at $1 / N$, which means that the initial polarization (local excitation defined by Eq. 5) is uniformly spread over the whole spin set. This is indeed the standard picture of decoherence process leading to an irreversible spread. But, if $\Delta$ is further increased, the LE-decay slows down showing long tails, see Fig. 1.(b). These destroy the saturation at least in the time-window analyzed in the present work. Such regime may be associated with a glassy polarization dynamics, i.e. the prevalence of the freezing effect of the Ising terms over the spreading induced by the $X Y$ ones.

If we consider $\Delta=0$ and let the disorder $h$ increase, the picture is indeed the standard Anderson localization problem. In such a case, the localization length must be compared to the finite size of the system. Hence, for very weak disorder, the LE degrades smoothly, as the localization length is longer than the system's size. When the disorder is strong enough, the localization length is smaller than the system's size and thus the initial local excitation remains around the site 1 . In fact, the crossover between these two physical situations can be quantified equating the system's size with the localization length $\lambda \simeq 24 J^{2} / h^{2}$, given in a FGR estimation ${ }^{27}$. This yields $h=\sqrt{2} J$, in fairly good agreement with the behavior shown in Figs. 1 (c) and (d).

Notice that in any case, neither Ising interactions nor Anderson disorder, can produce a LE-decay faster than a well defined time scale (see Figs. 1 (b) and $(\mathbf{d})$ ). This is specifically determined by the SP of the local excitation under the evolution given by $\hat{H}_{0}$,

$P_{1,1}^{0}(2 t)=2\left\langle\Psi_{n e q}\left|\exp \left(\mathrm{i} \hat{H}_{0} t / \hbar\right) \hat{S}_{1}^{z} \exp \left(-\mathrm{i} \hat{H}_{0} t / \hbar\right)\right| \Psi_{n e q}\right\rangle$,

where we emphasize that there is no dependence on $\hat{\Sigma}$. Such an $\hat{H}_{0}$-controlled decay resembles the perturbation independent decay experimentally observed in spin systems 21 and the Lyapunov regime of classically chaotic systems ${ }^{22}$.

In Fig. 2 we show the role of the time-scale determined by Eq. 7, acting as the limit for LE decay rates. These results are quite general, similar behavior is obtained for next nearest neighbors interactions (both in $\hat{H}_{0}$ and $\hat{\Sigma}$ ), provided that $\hat{\Sigma}$ has only Ising terms or Anderson disorder.

In order to analyze the ergodicity of the polarization dynamics observed in our finite system, we evaluate the mean LE, $\bar{M}_{1,1}$ :

$$
\bar{M}_{1,1}(T)=\frac{1}{T} \int_{0}^{T} M_{1,1}(t) d t .
$$

The standard analysis of the Anderson localization problem should imply, in the present case, to compute $\lim _{T \rightarrow \infty} \bar{M}_{1,1}(T)$. Instead, we analyze $\bar{M}_{1,1}(T)$ at $T=$ $12 \hbar / J$ which, as shown in Fig. 1, is long enough to allow for a uniform spreading of the polarization, provided that $\Delta$ is strong. Also, a rigorous upper bound for the

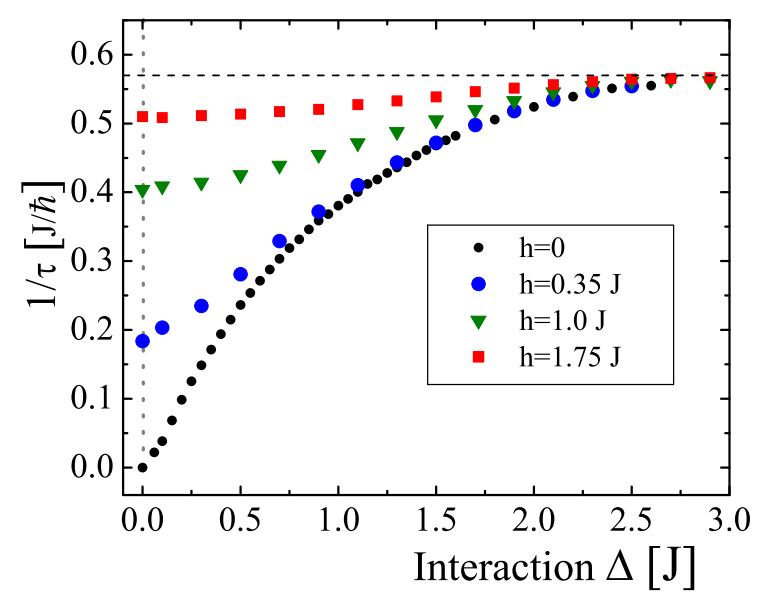

FIG. 2. Color online. LE decay rates as a function of the interaction strength $\Delta$, for different disorder magnitudes $h$. Horizontal dashed line represents the $\left(\hat{H}_{0}\right)$ SP time scale, i.e. Eq. 7. Data for $\Delta=0$ was obtained from single-particle physics and 500 disorder realizations, while data for $\Delta>0$ was obtained from full many-body simulations and 10 disorder realizations. The smoothness of matching evidences the robustness of the simulations performed.

integration time $T$ must be the system's Heisenberg time $T_{H}$, at which finite-size recurrences show up. In Fig. 3 we show a level plot of $\bar{M}_{1,1}$ as a function of the interaction $\Delta$ and disorder strength $h$. This results in a qualitative phase-diagram which evidences the competition between such physical magnitudes. When both $\Delta$ and $h$ are weak, $\bar{M}_{1,1}$ remains near 1 , since the system is almost reversible. Thus, the parametric region at the bottom left corner may be associated with decoherence, i.e. the system is weakly perturbed by uncontrolled degrees of freedom. If either $\Delta$ or $h$ are further increased, the system enters in a diffusive regime where the initial local polarization rapidly spreads irreversibly all across the spin system. Consistently, this bluish region is associated with an ergodic behavior for the polarization, since it is equally distributed along the spin system. For low disorder $(h \lesssim 0.5 J)$, increasing $\Delta$ leads to an Ising predominance, which freezes the polarization dynamics. We interpret such behavior as a glassy dynamics, with long relaxation times. This localization keeps $\bar{M}_{1,1}$ high. Analogously, increasing $h$ for a fixed value of $\Delta$ evidences a smooth crossover to a localized phase, where the polarization does not diffuse considerably.

Notice that localization by disorder is weakened when $1.0 J \lesssim \Delta \lesssim 2.0 J$, since the ergodic region seems to unfold for larger $h$. In fact, such values of interaction strength correspond to a faster arrival to the $1 / N$ saturation, as shown in Figs. 1 (a) and (b) (when $h=0$ ). Additionally, data around the $\Delta$ axis show that the disorder tends to abruptly destroy the quenching produced by Ising interactions. This seems suggestive of a parameter region where the interaction-disorder competition leads to a sharp transition between glassy and ergodic phases. 


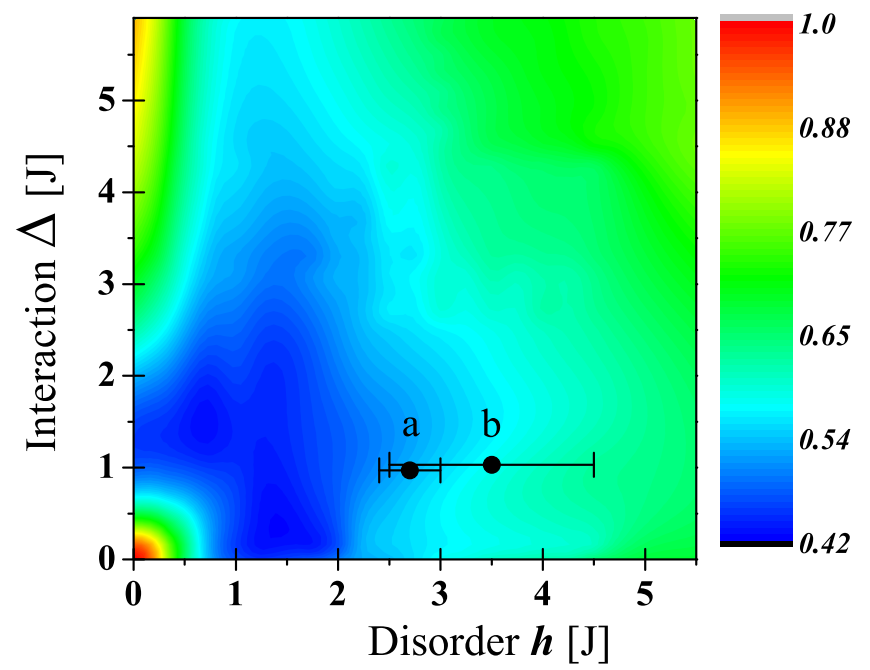

FIG. 3. Color online. Phase diagram for $\bar{M}_{1,1}(T)$ at $T=$ $12 \hbar / J$. Data point (a) is given for the MBL transition in Ref $^{28}, \Delta=1.0 J, h_{c}=(2.7 \pm 0.3) J$. Data point (b) is given for the MBL transition in Ref. $\stackrel{8}{ }, \Delta=1.0 \mathrm{~J}, h_{c}=(3.5 \pm 1.0) \mathrm{J}$. These points are slightly shifted in the plot from $\Delta=1.0 \mathrm{~J}$ in order to avoid their overlap.

However, a reliable finite size scaling of this regime would require excessively long times to capture how a vitreous dynamics is affected by disorder.

Previous numerical results of the $\mathrm{SP}^{28}$ and an analysis of the many-body eigenstates 8 of the same spin model have identified critical values for the MBL transition. Quite remarkably, they lie precisely at the crossover between the ergodic and the localized phases of the LE; see data-points (a) and (b) in Fig. 3. In our simulations, increasing $N$ (e.g. 10, 12 and 14) enables a larger integration time $T$, since $T \lesssim T_{H} \propto N$. In fact, when $\Delta \sim 1.0 \mathrm{~J}$, it can be verified that both sides of the tran- sition are well behaved since $\bar{M}_{1,1} \sim 1 / N$ in the ergodic regime, while $\bar{M}_{1,1} \sim 1 / \lambda$ for $h$ strong enough (regardless of $N$ ), and $d \bar{M}_{1,1} / d h$ increases with $N$. However, this finite size scaling of $\bar{M}_{1,1}(T)$ within our accessible range is not enough to yield precise critical values for the MBL transition.

In summary, we were able to draw a qualitative phasediagram for the polarization under the LE dynamics, identifying ergodic, localized and glassy regimes. It displays a nontrivial geography with a deep penetration of the ergodic phase into the glassy domain separating it from the localized region. Besides, while in finite 1-d systems Anderson localization is indeed a smooth crossover, it seems to develop into a ergodic-localized transition for nonzero interactions. Additionally, our results suggest that the glassy-ergodic transition is a better candidate for a sharp phase transition. In spite of the fact that the local nature of the observable constitutes a limitation to perform a reliable finite size scaling, our strategy seems promising to analyze different underlying topologies and different ways to breakdown integrability. Last, but not least, in state-of-the-art nuclear magnetic resonance ${ }^{29,30}$, the high temperature correlation functions, like the LE, are recognized witnesses for the onset of phase transitions 31 , even hinting at the appearance of many-body localization ${ }^{32,33}$.

P.R.Z., A.D.D. and H.M.P. wish to dedicate this paper to the memory of their coauthor P.R.L. who did not live to see the final version of this paper. This work benefited from discussions with L.F. Santos, and comments by O. Osenda and F. Pastawski. P.R.Z. and H.M.P. acknowledge M.C. Bañuls and J.I. Cirac for their kind hospitality at MPQ in Garching. We acknowledge support from CONICET, ANPCyT, SeCyT-UNC and MinCyTCor. The calculations were done on Graphical Processing Units under a NVIDIA Professor Partnership Program led by O. Reula.
1 E. Fermi, J. Pasta, and S. Ulam, LASL Report LA1940 5, 977 (1955).

2 G. P. Berman and F. M. Izrailev, Chaos 15, 015104 (2005)

3 C. Gogolin, M. P. Müller, Phys. Rev. Lett. 106, 040401 (2011)

4 A. Polkovnikov, K. Sengupta, A. Silva, and M. Vengalattore, Rev. Mod. Phys. 83, 863 (2011)

5 S. Goldstein, J. L. Lebowitz, R. Tumulka, and N. Zanghì, Eur. Phys. J. H 35, 173 (2010) arXiv:1003.2129 [quant-ph].

6 T. Kinoshita, T. Wenger, and D. S. Weiss, Nature 440, 900 (2006) S. Trotzky, Y.-A. Chen, A. Flesch, I. P. McCulloch, U. Schollwöck, J. Eisert, and I. Bloch, Nat. Phys. 8, 325 (2012) arXiv:1101.2659 [cond-mat.quant-gas]

7 D. M. Basko, I. L. Aleiner, and B. L. Altshuler, Ann. Phys. 321, 1126 (2006), arXiv:cond-mat/0506617.

8 A. Pal and D. A. Huse, Phys. Rev. B 82, 174411 (2010).
${ }^{9} \mathrm{~V}$. Oganesyan and D. A. Huse, Phys. Rev. B 75, 155111 (2007).

10 P. W. Anderson, Rev. Mod. Phys. 50, 191 (1978)

11 S. Popescu, A. J. Short, and A. Winter, Nat. Phys. 2, 754 (2006).

12 M. Rigol, V. Dunjko, and M. Olshanii, Nature 452, 854 (2008), arXiv:0708.1324 [cond-mat.stat-mech].

13 M. Z̈nidarič, T. Prosen, and P. Prelovšek, Phys. Rev. B 77, 064426 (2008).

14 J. H. Bardarson, F. Pollmann, and J. E. Moore, Phys. Rev. Lett. 109, 017202 (2012).

15 I. L. Aleiner, B. L. Altshuler, and G. V. Shlyapnikov, Nat. Phys. 6, 900 (2010), arXiv:0910.4534 [cond-mat.dis-nn].

$16 \mathrm{~V}$. V. Flambaum and F. M. Izrailev, Phys. Rev. E 64, 036220 (2001)

17 V. V. Flambaum and F. M. Izrailev, Phys. Rev. E 64, 026124 (2001) 
18 C. Neuenhahn and F. Marquardt, Phys. Rev. E 85, 060101 (2012).

19 L. F. Santos, F. Borgonovi, and F. M. Izrailev, Phys. Rev. Lett. 108, 094102 (2012).

20 T. Gorin, T. Prosen, T. H. Seligman, and M. Žnidarič, Phys. Rep. 435, 33 (2006) P. Jacquod and C. Petitjean, Adv. in Phys. 58, 67 (2009) D. Wisniacki, A. Goussev, R. Jalabert, and H. Pastawski, Scholarpedia 7, 11687 (2012).

21 P. R. Levstein, G. Usaj, and H. M. Pastawski, J. Chem. Phys. 108, 2718 (1998)

22 R. A. Jalabert and H. M. Pastawski, Phys. Rev. Lett. 86, 2490 (2001)

23 P. R. Zangara, A. D. Dente, P. R. Levstein, and H. M. Pastawski, Phys. Rev. A 86, 012322 (2012).

24 E. P. Danieli, H. M. Pastawski, and P. R. Levstein, Chem. Phys. Lett. 384, 306 (2004).

25 G. A. Álvarez, E. P. Danieli, P. R. Levstein, and H. M. Pastawski, Phys. Rev. Lett. 101, 120503 (2008)

26 A. D. Dente, C. S. Bederián, P. R. Zangara, and H. M. Pastawski, ArXiv e-prints (2013), arXiv:1305.0036 [physics.comp-ph]

27 B. Kramer and A. Mackinnon, Rep. Prog. Phys. 56, 1469 (1993)

28 A. D. Luca and A. Scardicchio, Europhys. Lett. 101, 37003 (2013).

29 M. B. Franzoni, R. H. Acosta, H. M. Pastawski, and P. R. Levstein, Philos. T. Roy. Soc. A 370, 4713 (2012).

30 S. W. Morgan, V. Oganesyan, and G. S. Boutis, Phys. Rev. B 86, 214410 (2012).

31 J. Zhang, F. M. Cucchietti, C. M. Chandrashekar, M. Laforest, C. A. Ryan, M. Ditty, A. Hubbard, J. K. Gamble, and R. Laflamme, Phys. Rev. A 79, 012305 (2009)

32 M. B. Franzoni and P. R. Levstein, Phys. Rev. B 72, 235410 (2005)

33 G. A. Álvarez and D. Suter, Phys. Rev. Lett. 104, 230403 (2010) 